Int. J. Odontostomat., 7(2):207-213, 2013.

\title{
Estomatitis Subprotésica en Pacientes de la IX Región, Chile
}

\author{
Denture Stomatitis Present in Patients of the IX Region, Chile
}

Carmen G. Gutiérrez; Luis Bustos Medina**; Marcela Sanchez ${ }^{\star * *}$; Luis Zaror Cornejo ${ }^{\star * \star}$ \& María E. Zambrano ${ }^{\star * * * *}$

GUTIÉRREZ, F. C. G.; BUSTOS, M. L.; SÁNCHEZ, M.; ZAROR, C. L. \& ZAMBRANO, M. E. Estomatitis Subprotésica en pacientes de la IX región, Chile. Int. J. Odontostomat., 7(2):207-213, 2013.

RESUMEN: El objetivo de este estudio, fue determinar la prevalencia de estomatitis subprotésica y sus factores asociados en pacientes portadores de prótesis removibles. Estos pacientes pertenecieron al Departamento de Prostodoncia del Consultorio Miraflores y a la Clínica Odontológica de la Universidad Mayor entre Septiembre 2009 y Diciembre 2010. El total de pacientes examinados fueron 75 portadores de prótesis removibles. Se tomaron dos muestras a cada paciente, una proveniente del paladar y otra de su prótesis, con su posterior estudio microbiológico. El $68 \%$ de los pacientes presentó alteraciones en la mucosa palatina compatibles con estomatitis subprotésica. En el $74,6 \%$ de los pacientes con estomatitis subprotésica se aisló al menos una especie de Candida, no existiendo diferencia significativa. La prótesis dental removible influye notablemente en la aparición de la estomatitis subprotésica. Al aumentar la gravedad de la enfermedad, aumenta la presencia de Candida, siendo Candida albicans la más prevalente en pacientes con estomatitis subprotésica.

PALABRAS CLAVE: estomatitis subprotésica, Candida, prótesis removible, Candida albicans.

\section{INTRODUCCIÓN}

El proceso de envejecimiento es inexorable e irreversible. Modifica los tejidos, órganos y sistemas, y a medida que el individuo aumenta en edad, afectando la autoestima, autovalencia, sus relaciones interpersonales y afectivas (Misrachi et al., 1995). En este contexto, la cavidad oral es parte del organismo y forma el sistema estomatognático.

Los tejidos orales y periorales no escapan al proceso de envejecimiento, observándose en ellos diversos cambios, como: pérdida de dientes, disminución del tejido óseo de la mandíbula y de los maxilares y disminución del flujo salival, entre otros (OPS/ OMS, 2003).

Diversos estudios (Adulyanon et al., 1996; Appollonio et al., 1997; Chen \& Hunter, 1996; Gift et al., 1997; Misrachi et al., 2002) han relacionado el es- tado de salud oral, con la calidad de vida del adulto; coincidiendo algunos en afirmar que para éstos es importante su salud oral. Sin embargo, en la población chilena, según la última Encuesta Nacional de Salud, sólo un $27,8 \%$ de la población tiene dentadura completa y los desdentados totales empiezan a ser frecuentes en el grupo mayor de 65 años, alcanzando una prevalencia de $33,4 \%$ al considerar mandíbula y maxilares (Gamonal, 1996; MINSAL, 2003; Kressin et al., 1996).

Su rehabilitación oral incluye, para la gran mayoría, la confección de prótesis dentales removibles, parciales o totales, para reemplazar un tratamiento previo en malas condiciones o para sustituir los dientes perdidos por enfermedad periodontal o caries causadas por los deficientes conocimientos acerca de higiene oral y protésica (Marin, 1993; OPS/OMS).

Departamento de Odontología Universidad Mayor, Temuco, Chile.

* Centro de Excelencia CIGES (Capacitación, Investigación y Gestión para la Salud Basada en la Evidencia). Dpto. Salud Pública. Facultad de Medicina, Universidad de La Frontera, Temuco, Chile.

*** Departamento de Tecnología Médica Universidad Mayor, Temuco, Chile.

**** Departamento de Prostodoncia del Consultorio Miraflores, Temuco-Chile. Investigación financiada en parte por el Fondo de Investigación y Desarrollo de la Universidad Mayor (FIDUM): Candida sp. en estomatitis subprotésica en pacientes con prótesis dental removible, sus factores de patogenicidad. 
GUTIÉRREZ, F. C. G.; BUSTOS, M. L.; SÁNCHEZ, M.; ZAROR, C. L. \& ZAMBRANO, M. E. Estomatitis Subprotésica en pacientes de la IX región, Chile. Int. J. Odontostomat., 7(2):207-213, 2013

Sólo en el año 2002, se realizaron en los servicios públicos del país 18.245 prótesis removibles (MINSAL, 2013). Algunas lesiones originadas por prótesis dental son las lesiones erosivo-ulcerosas. La estomatitis subprotésica es una de ellas, alta prevalencia y multifactorial, lo que hace más complejo su estudio y, aún más, su pronóstico (Lazarde \& Pacheco, 2001).

En Chile, son escasos los estudios descritos, que permitan conocer la prevalencia de estomatitis subprotésica en los pacientes portadores de prótesis dental removible y las especies Candida involucradas, en esta patología (Espinoza et al., 2003). El propósito de éste estudio es contribuir a comprender mejor la estomatitis subprotésica y darle una mejor orientación etiológica, además de contribuir a su mejor manejo clínico.

\section{MATERIAL Y MÉTODO}

Estudio de corte transversal en 75 pacientes que acudieron al Servicio de Odontología de la clínica de prótesis del Consultorio Miraflores y a la Clínica Odontológica de la Universidad Mayor durante los años 2009 y 2010.

Los pacientes fueron previamente informados de las características de la investigación y firmaron un consentimiento escrito, aceptando participar del proyecto.

Se confeccionó una ficha clínica, que proporcionó nombre, edad, RUT, fecha de nacimiento y procedencia. La información correspondiente al tipo y material usado en la confección de las prótesis dentales se obtuvo a través de la observación directa de las mismas.

Al efectuar el interrogatorio se respondió a variables como: condición sistémica, presencia de hábitos nocivos en el paciente (tabaquismo, alcoholismo). En cuanto a la prótesis dental, se respondió a interrogantes como: tiempo de uso de la última prótesis, sensación de flujo bucal disminuido, $\mathrm{pH}$ salival en el momento de la toma de muestra, tipo de prótesis y material de la base, procedimiento de descontaminación de la prótesis,adaptación de la prótesis a la mucosa oral, síntomas de estomatitis subprotésica y uso nocturno de la prótesis.

El examen clínico intraoral se realizó para evidenciar la presencia de estomatitis subprotésica. Se clasificó la patología en tipos I, II o III, según los crite- rios de Newton (1962). Se especificó el área del paladar duro afectada. De cada paciente, se obtuvo dos muestras a fin de determinar la presencia de especies del género Candida.

La Muestra 1 se obtuvo directamente de la mucosa palatina del paladar duro mediante una tórula estéril. Esta fue almacenada en un tubo de vidrio con solución Ringer, para impedir que Candida sp. perdiera viabilidad en el algodón seco. La Muestra 2 se obtuvo de la superficie interna de la prótesis, que tomaba contacto con la mucosa palatina, empleando para ello un bisturí estéril con el que se raspó la superficie protésica, preferentemente en aquellas zonas con evidente acumulación de residuos. Lo obtenido con el bisturí se recoge con una tórula estéril contenida en un tubo de vidrio con solución Riger.

Las muestras se mantuvieron a temperatura ambiente, durante algunas horas, hasta su siembra en el Laboratorio de Microbiología Clínica de la Universidad Mayor. Posteriormente, se efectuó la siembra de las muestras de prótesis y paladar en agar Sabouraud y CHROMagar Candida, frotando las tórulas en la superficie del medio y extendiendo el inóculo con la ayuda de un asa de nicrón esterilizada al mechero. Los cultivos fueron incubados a $37^{\circ} \mathrm{C}$ en condiciones de aerobiosis durante 48 horas. En forma conjunta, se efectúo un frotis Gram directo de las muestras de prótesis y paladar, para evidenciar la presencia de levaduras a priori (Fig. 1).

Para la identificación de Candida albicans, se realizaron tres pruebas que permitieron discriminar a esta especie del resto de levaduras del mismo género encontradas. 1. Efecto de Reynold - Braude: la formación de túbulo germinativo se presenta en Candida albicans y Candida dubliniensis. 2. Crecimiento en agar Sabouraud dextrosa a $42^{\circ} \mathrm{C}$ : Los cultivos que presentaron túbulo germinativo fueron incubados en agar Sabouraud dextrosa, a 37 y $42^{\circ} \mathrm{C}$ durante $48 \mathrm{~h}$. Sólo Candida albicans crece a $42^{\circ} \mathrm{C}$, diferenciándose de Candida dubliniensis (Ballesté et al., 2005). 3. Crecimiento en solución de $\mathrm{NaCl}$ al $6,5 \%$ : Los cultivos que presentaron túbulo germinativo también fueron incubados en solución salina al $6,5 \%$, a $37^{\circ} \mathrm{C}$ durante 48 h. Sólo Candida albicans crece en estas condiciones.

Las cepas que no desarrollaron en agar Sabouraud dextrosa a $42^{\circ} \mathrm{C}$, fueron sembradas en agar Tabaco. Las colonias de Candida dubliniensis, producen colonias ásperas de color marrón-amarillentas después de su incubación a $28^{\circ} \mathrm{C}$ por $48-72 \mathrm{~h}$. 
La identificación de las levaduras que no eran Candida albicans, se realizó a través de: API 20 C AUX (bioMérieux) y RapID Yeast Plus System (Remel).

Se realizó análisis univariado y bivariado para determinar la asociación entre las variables empleando la prueba chi-cuadrado y la prueba exacta de Fisher. El procesamiento y análisis de los datos se realizó con el programa estadístico STATA, versión 11.0.

\section{RESULTADOS Y DISCUSIÓN}

De los 75 pacientes portadores de prótesis, la mayoría estuvo conformado por mujeres, en un $80 \%$, cuyas edades estuvieron comprendidas entre 33 y 75 años de edad, con un promedio de 60,2 años con DE 12,11 (Fig. 2).

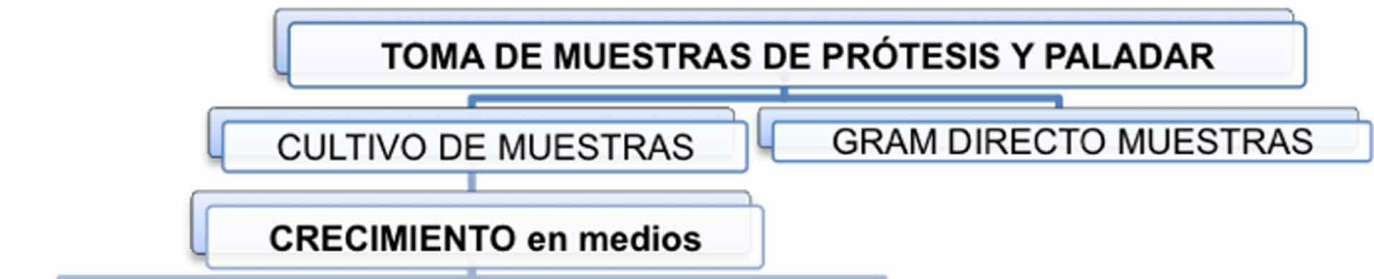

$(-)$

No hay aislamiento de Candida sp

Cultivo de aislamientos En CHROMagar Candida.

Cultivo de aislamientos en Agar Sabouraud.

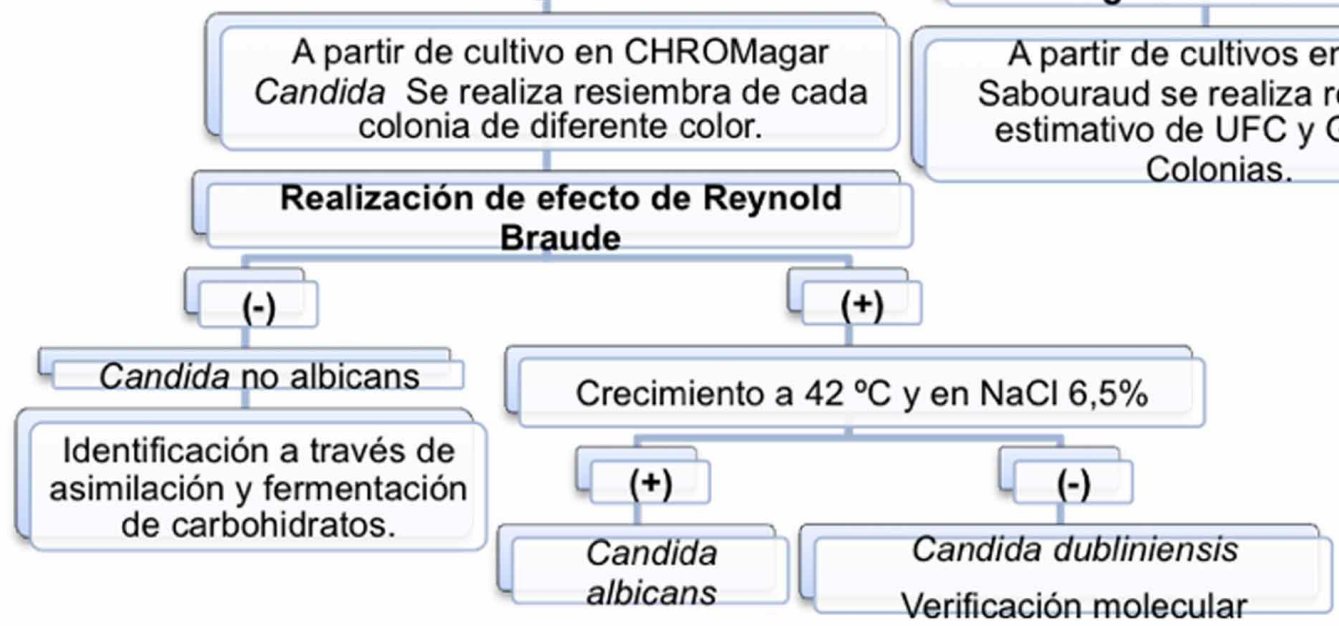

Fig. 1. Flujograma de estudio de estomatitis subprotésica.

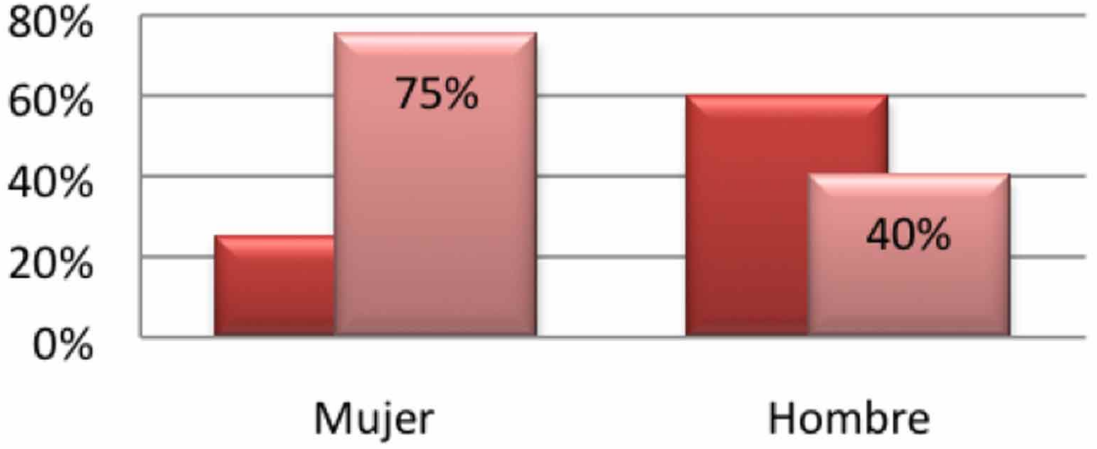

Fig. 2. Distribución de pacientes con estomatitis subprotésica según sexo.
Ausencia de Estomatitis Subprotésica

Presencia de Estomatitis Subprotésica

Fisher $p=0,014$ 
El $68 \%$ de los pacientes del grupo de estudio, desarrolló algún tipo de estomatitis subprotésica (intervalo de confianza de 57 a $79 \%$ ). De acuerdo a la clasificación de Newton, el $47 \%$ de los pacientes presentó estomatitis subprotésica tipo I, el $25,5 \%$ tipo II y $27,5 \%$ tipo III. El porcentaje de pacientes que padecían de estomatitis subprotésica, superó al porcentaje nacional registrado, que corresponde al 22,3\% (Espinoza et al.). Esto se debe principalmente a la población estudiada, provenientes de sectores rurales y alejados de centros de salud oral especializados. Estos resultados coinciden con los reportados en otros estudios realizados, la cual va desde $43 \%$ hasta $70 \%$ (Gendreau \& Loewy, 2011; Nápoles et al., 2009).

La Figura 2 muestra que, el $75 \%$ de las mujeres que participaron en el estudio, presentó estomatitis subprotésica. Existió evidencia estadísticamente significativa para concluir que los porcentajes entre hombres y mujeres son distintos. Muy semejante a otras publicaciones (Ley et al., 2010; Nápoles et al., 2009). Esto puede deberse a la supuesta tendencia de las mujeres a perder los dientes más tempranamente y a que acudirían con más frecuencia que los hombres a la consulta odontológica para resolver sus problemas dentales (Dorey et al., 1985; Cardozo et al., 2001).

En la Figura 3, el 77,4\% de los pacientes portadores de prótesis por igual o más de 6 años desarrollaron estomatitis subprotésica, existiendo evidencia estadísticamente significativa para concluir que los porcentajes para el tiempo de uso de la última prótesis son distintos. En este sentido, los resultados indican que a medida que aumenta el tiempo de uso, aumenta la presencia de estomatitis, así como su severidad (Nápoles et al.).
La Tabla I, muestra que el $76,9 \%$ de los pacientes que portaban prótesis totales superiores y el $47,8 \%$ de los usuarios de prótesis parciales superiores, desarrollaron estomatitis subprotésica. El 73,8\% de los pacientes que portaban prótesis acrílicas presentaron estomatitis subprotésica, existiendo diferencia estadísticamente significativa para concluir que los porcentajes tanto para el tipo de prótesis como el material de la base, son distintos. Resultados similares obtuvo Lazarde \& Pacheco (2001), quienes explican que en pacientes portadores prótesis parciales, existen dientes remanentes, por lo que la prótesis presentan una mejor biomecánica y el paciente debe extremar su medidas higiénicas, para evitar pérdida de piezas dentarias (Moreira et al., 1989). Varios autores concuerdan (Ley et al.) y explican que la porosidad del acrílico de la prótesis, favorece la adhesión de Candida por producción de biofilm, constituyéndose la prótesis en un reservorio para causar este tipo de lesión (Dorey et al.; Moreira et al.).

En cuanto al uso nocturno de la prótesis (Tabla II), de los pacientes que relataron dormir con su prótesis, el $25,5 \%$ no presentó la enfermedad. En contraste, el $74,5 \%$ presentaron estomatitis subprotésica, lo que fue estadísticamente significativo. Los datos coinciden con Carreira \& Almagro (2000); quienes comentan que el uso de prótesis por 24 horas sin descansar, provoca la degeneración de las glándulas salivales y disminuye el flujo salival, lo cual agrava más la lesión.

En la Tabla III se observa, que en $77,1 \%$ de los pacientes con estomatitis subprotésica se aisló al menos una especie de Candida. Esta diferencia, no es significativa desde el punto de vista estadístico. Estos

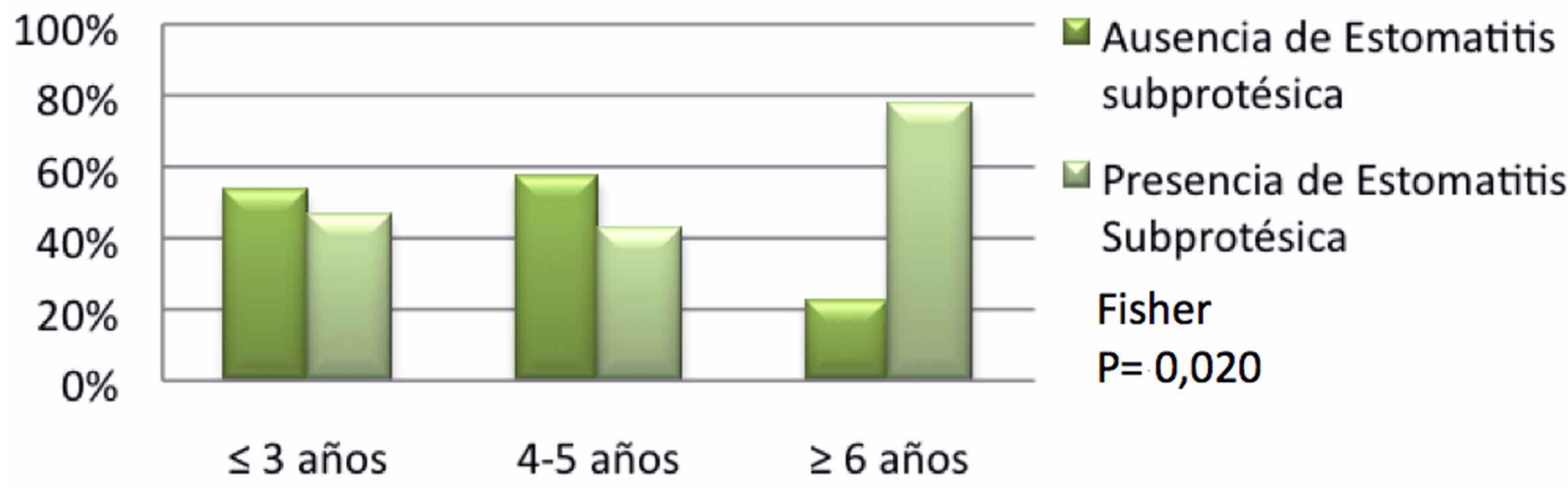

Fig. 3. Distribución de pacientes según el tiempo de uso de la última prótesis. 
GUTIÉRREZ, F. C. G.; BUSTOS, M. L.; SÁNCHEZ, M.; ZAROR, C. L. \& ZAMBRANO, M. E. Estomatitis Subprotésica en pacientes de la IX región, Chile. Int. J. Odontostomat., 7(2):207-213, 2013.

Tabla I. Distribución de pacientes del estudio según el tipo de prótesis y el tipo de base protésica.

\begin{tabular}{|c|c|c|c|c|c|c|}
\hline & \multicolumn{4}{|c|}{ Estomatitis subprotésica } & \multirow[b]{3}{*}{ Total } & \\
\hline & \multicolumn{2}{|c|}{ Ausente } & \multicolumn{2}{|c|}{ Ausente } & & \\
\hline & Número & $\%$ & Número & $\%$ & & \\
\hline Prótesis Total superior & 12 & 23,1 & 40 & 76,9 & 52 & \multirow{3}{*}{$\mathrm{p}=0,017 \mathrm{Chi}^{2}$} \\
\hline Prótesis Parcial superior & 12 & 52,2 & 11 & 47,8 & 23 & \\
\hline Base Acrílica & 17 & 26,2 & 48 & 73,8 & 65 & \\
\hline Base Metálica & 6 & 66,7 & 3 & 33,3 & 9 & \multirow[t]{2}{*}{$p=0,010$ Fisher } \\
\hline Base Mixta & 1 & 100 & 0 & 0 & 1 & \\
\hline
\end{tabular}

Tabla II. Distribución de pacientes con estomatitis subprotésica según hábito nocturno de uso de la prótesis.

\begin{tabular}{lcccccc}
\hline & \multicolumn{3}{c}{ Estomatitis subprotésica } & & \\
\cline { 2 - 5 } & \multicolumn{2}{c}{ Ausente } & \multicolumn{2}{c}{ Ausente } & & \\
\cline { 2 - 5 } Número & $\%$ & Número & $\%$ & Total & \\
\cline { 2 - 5 } No duerme con prótesis & 10 & 50 & 10 & 50 & 20 & p $=0,044$ Ch́ \\
\cline { 2 - 5 } Duerme con la prótesis & 14 & 25,5 & 41 & 74,5 & 55 & \\
\hline
\end{tabular}

Tabla III. Distribución de pacientes de estudio según cultivo de levaduras del género Candida.

\begin{tabular}{lcccccc}
\hline & \multicolumn{3}{c}{ Estomatitis subprotésica } & & \\
\cline { 2 - 5 } & \multicolumn{3}{c}{ Ausente } & Ausente & & \\
\cline { 2 - 5 } & Número & $\%$ & Número & $\%$ & Total & \\
\cline { 2 - 6 } Cultivo Negativo & 16 & 40 & 24 & 60 & 40 & $\mathrm{p}=0,140 \mathrm{Chi}^{2}$ \\
Cultivo Positivo & 8 & 22,9 & 27 & 77,1 & 35 & \\
\hline
\end{tabular}

Tabla IV. Distribución de la severidad clínica de los pacientes con estomatitis según la presencia y número de aislamientos de especies Candida.

\begin{tabular}{lllllllll} 
& \multicolumn{9}{l}{ Estomatitis Subprotésica } \\
\cline { 2 - 8 } & \multicolumn{2}{l}{ ESP tipo I } & ESP tipo II & ESP tipo III & Total & \\
\cline { 2 - 8 } Cultivo negativo & 14 & $70 \%$ & 4 & $20 \%$ & 2 & $10 \%$ & 20 & $\mathrm{p}=0,02 \mathrm{Chi}^{2}$ \\
Cultivo positivo & 10 & $32,3 \%$ & 9 & $29 \%$ & 12 & $38,7 \%$ & 31 & \\
\hline
\end{tabular}

datos se contraponen a los de Brevis et al., que demuestran que la presencia de Candida cumple un rol fundamental en el desarrollo de la estomatitis subprotésica. Sin embargo, el alto porcentaje de pacientes con estomatitis subprotésica y cultivo negativo, $60 \%$, muestra que esta lesión puede ser causada por otros factores que no impliquen necesariamente la presencia de hongos. El 22,9\% de los pacientes portadores de prótesis removible sin estomatitis subprotésica presentaron levaduras al cultivo. Ello se explica porque Candida es un comensal habitual de la cavidad oral (Ottonelli et al., 2012; Brevis et al., 2008).
La Tabla IV muestra, que el $38,7 \%$ de los pacientes que se clasificó en el examen clínico como estomatitis subprotésica tipo III según Newton, al examen micológico presentó, cultivo positivo para especies de Candida. En cambio, para la estomatitis subprotésica tipo I, en el $70 \%$ no hubo desarrollo, lo que es estadísticamente significativo, según el tipo de estomatitis. Al aumentar la gravedad de la enfermedad, aumenta la presencia de Candida, siendo Candida albicans la más prevalente en estos pacientes (Brevis et al.; Mata De Henning \& Perrone, 2001; Mathaba et al., 1995). 
La Figura 4 muestra la distribución de especies de Candida encontradas en la prótesis de los pacientes con estomatitis subprotésica. Candida albicans representó al $73 \%$ de los aislamientos. Son numerosos los estudios que relacionan a la Candida albicans con la estomatitis subprotésica (Brevis et al.) esto tiene que ver con la capacidad de adherencia a través de ligandos específicos que actúan sobre las células epiteliales de la mucosa palatina.

Como se puede observar en la Tabla V, existe una mayor eficiencia de aislamiento e identificación de especies de género Candida, con la utilización del medio CHROMagar (100\%). Resultados similares obtuvo López et al. (2005). Ballesté et al. (2005) señalan, que el CHROMagar Candida tiene un buen desempeño diagnóstico para la identificación de Candida albicans (100\%), lo que se refleja en la elevada sensibilidad y especificidad. Sin embargo, al analizar el desempeño diagnóstico del medio para la identificación de cepas de Candida no albicans, éste mantiene una elevada sensibilidad perdiendo especificidad diagnóstica, por lo que se utilizan pruebas de asimilación para confirmar el diagnóstico. Su utilidad es allí orientadora.
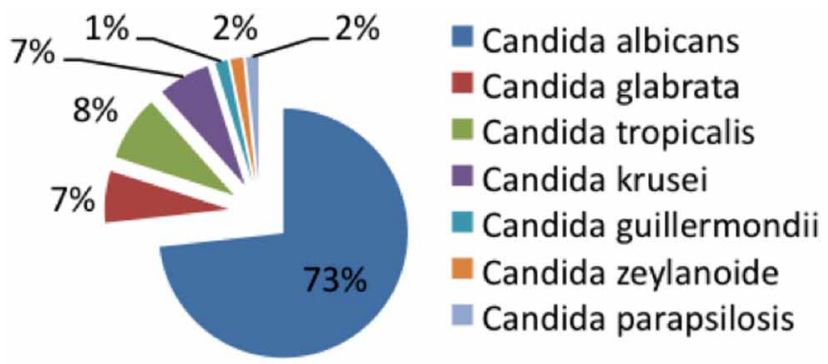

Fig. 4. Distribución de especies del género Candida aisladas tanto en prótesis como en paladar de pacientes con estomatitis subprotésica.

Tabla V. Rendimiento de los medios utilizados en el aislamiento de Candida sp. en pacientes con prótesis dental.

\begin{tabular}{lcc}
\hline Medios utilizados para aislamiento & $\mathrm{n}$ & $\%$ \\
\hline Aislamientos con agar Sabouraud dextrosa & 44 & 74,5 \\
Aislamientos con CHROMagar Candida & 59 & 100
\end{tabular}

GUTIÉRREZ, F. C. G.; BUSTOS, M. L.; SÁNCHEZ, M.; ZAROR, C. L. \& ZAMBRANO, M. E. Denture stomatitis present in patients of the IX region, Chile. Int. J. Odontostomat., 7(2):207-213, 2013.

ABSTRACT: In the present study, we determined the prevalence of denture stomatitis and its associated factors in patients that use removable denture. These patients come from the prosthetics clinic of the Miraflores Clinic and from the dental clinic of the Universidad Mayor between September 2009 and December 2010. The total of 75 patients with removable dentures were examined, two samples were taken of each patient, one of their palate and other of their prostheses, for performing microbiological studies. The $68 \%$ of the patients showed alterations in the palate mucosa subprotesic compatible with stomatitis. $74.6 \%$ of patients with denture stomatitis isolated at least one species of Candida, with no meaningful evidence. Removable dental prosthesis substantially affects the appearance of denture stomatitis. When the severity of illness increases the presence of Candida also increases, Candida albicans being the most prevalent in patients with denture stomatitis.

KEY WORDS: denture stomatitis, Candida, removable dentures, Candida albicans.

\section{REFERENCIAS BIBLIOGRÁFICAS}

Adulyanon, S.; Vourapukjaru, J. \& Sheiham, A. Oral impacts affecting daily performance in a low dental disease Thai population. Community Dent. Oral Epidemiol., 24(6):3859, 1996.

Appollonio, I.; Caraballese, C.; Frattola, A. \& Trabucchi, M. Dental status, quality of life, and mortality in an older community population: A multivariate approach. J. Am. Geriatr. Soc., 45(11):1315-23, 1997.

Ballesté, R.; Arteta, Z.; Fernández, N.; Mier, C.; Mousqués, N.; Xavier, B.; et al. Evaluación del medio cromógeno CHROMagar CandidaTM para la identificación de levaduras de interés médico. Rev. Méd. Urug., 21(3):18693, 2005.

Brevis, A. P.; Cancino, M. J. \& Cantín, L. M. Estomatitis subprótesis: Estudio clínico y microbiológico de Candida. Int. J. Odontostomat., 2(1):101-8, 2008.

Cardozo, E.; Salazar, E.; Perrone, M. \& Pardi, G. Detección de Candida albicans en pacientes con estomatitis subprotésica, medicados con anfotericina tópica. Acta Odontol. Venez., 41(3):188-94, 2003.

Carreira, P. V. \& Almagro, U. Z. La estomatitis subprótesis en pacientes desdentados totales. Rev. Cubana Estomatol., 37(3):133-9, 2000.

Chen, M. S. \& Hunter, P. Oral health and quality of life in New Zealand: a social perspective. Soc. Sci. Med., 43(8):1213-22, 1996.

Dorey, J. L.; Blasberg, B.; MacEntee, M. \& Conklin, R. Oral mucosal disorders in denture wearers. J. Prosthet. Dent., 53(2):210-3, 1985. 
Espinoza, I.; Rojas, R.; Aranda, W.; Gamonal, J. Prevalence of oral mucosal lesions in elderly in Santiago Chile. J. Oral Pathol. Med., 32(10):571-5, 2003.

Gamonal, J. Prevalencia de enfermedades periodontales y de caries dental en la población de 35-44 y de 65 a 74 años de nivel socioeconómico bajo y medio-bajo de la provincia de Santiago, Región Metropolitana, y determinación de los recursos humanos necesarios para su tratamiento. Rev. Fac. Odontol. Univ. Chile, 14(1):56-7, 1996.

Garcia, A. B.; Benet, R. M. \& Castillo, B. E. E. Prótesis dentales y lesiones mucosas en el adulto mayor. MediSur, 8(1):36-41, 2010.

Gendreau, L. \& Loewy, Z. G. Epidemiology and etiology of denture stomatitis. J. Prosthodont., 20(4):251-60, 2011.

Gift, H. C.; Atchinson K. A. \& Dayton, C.M. Conceptualizing oral health and oral health-related quality of life. Soc. Sci. Med., 44(5):601-8, 1997.

Kressin, N.; Spiro, A. 3rd.; Bosse, R.; García, R. \& Kazis, L. Assesing oral health related quality of life: Findings from the normative aging study. Med. Care, 34(5):416-27, 1996.

Lazarde, L. J. \& Pacheco, A. Identificación de especies de Candida en un grupo de pacientes con candidiasis atrófica crónica. Acta Odontol. Venez., 39(1):13-8, 2001.

Ley, L.; Martínez, S.; Puig, E.; Nápoles, I. \& Díaz, S. Comportamiento de la estomatitis subprotésis. AMC, 14(1):00, 2010.

López, C.; Giro, L.; Ramos, L.; Ramadán, S. \& Bulacio, L. Comparación de diferentes métodos para la identificación de especies del género Candida. Rev. Argent. Microbiol., 37(1):16-21, 2005.

Marin, P. Tiempo Nuevo para el Adulto Mayor: Enfoque interdisciplinario. Santiago, Universidad Católica de Chile ediciones, 1993. pp.12-13, 167-70.

Mathaba, L. T.; Davies, G. \& Warmington, J. R. The genotypic relationship of Candida albicans strains isolated from the oral cavity of patients with denture stomatitis. J. Med. Microbiol., 42(5):372-9, 1995.

Mata De Henning, M. \& Perrone, M. La Prótesis Odontológica en la Ecología de Candida albicans en Cavidad Bucal. Acta Odontol. Venez., 39(3):18-24, 2001.

MINSAL. Departamento de Estadística e información en salud, 2013. Disponible en http://deis.minsal.cl.

MINSAL. Resultados I Encuesta Nacional de Salud. Informe Final. Santiago, Chile, Ministerio de Salud, 2003. pp.340, 344. Disponible en: http://epi.minsal.cl/Epi/Html/ Invest/ENS/InformeFinalENS.pdf

Misrachi, L.; Cavargas, J.; Biotti, J. \& Soto, R. La salud oral y el adulto mayor, desafíos para la Odontología. Rev. Fac. Odontol. Univ. Chile, 13(1):9-15, 1995.

Misrachi, C.; Sepulveda, H. \& Lamadrid, S. Situación protésica y conductas asociadas en adultos mayores de nivel socioeconómico medio-alto y Bajo. Rev. Dent. Chile, 9381:10-6, 2002.

Moreira, E.; Bernal, A.; Urbizo, J. \& Molina, J. Estomatitis Subprotésica: Estudio epidemiológico en 6302 pacientes portadores de prótesis dentales removibles. Rev. Cubana Estomatol., 26(1-2):71-80, 1989.

Nápoles, I.; Diaz, S.; Puig, E. \& Casanova, Y. Prevalencia de la estomatitis subprótesis. AMC,13(1):0-0, 2009.

Newton, A. V. Denture sore mouth: a possible aetiology. $\mathrm{Br}$. Dent. J., 112:357-60, 1962.

OPS/OMS. Guía clínica para atención primaria a las personas adultas mayores. ( $3^{a}$ ed.). Washington D.C., Organización Panamericana de la Salud/Organización Mundial de la Salud, 2003.

Ottonelli, S. C. D.; Mezzomo, C. F.; Aulo, O. F.; Piva, E.; Borges, G.; Werner, S. S. M.; et al. Antimicrobial activity of [2-(methacryloyloxy)ethyl]trimethylammonium chloride against Candida spp. Rev. Iberoam. Micol., 29(1):20-3, 2012.

Dirección para Correspondencia:

TM. Dr. Sc. Luis Zaror Cornejo

Profesor Titular de Microbiología

Departamento de Tecnología Médica

Universidad Mayor

Temuco

CHILE

Email: Izaror@yahoo.es

Recibido : 24-10-2012

Aceptado: 07-05-2013 